\title{
Colposcopic Evaluation of Pre and Postmenopausal Women with Abnormal Cervical Cytologies
}

\author{
Keziban Doğan ${ }^{1}$, Hakan Güraslan ${ }^{1}$ \\ ${ }^{1}$ Department of Obstetrics and Gynecology, Bakirkoy Dr Sadi Konuk Teaching and Research Hospital, Istanbul/Turkey
}

Received: 17 March 2016, Accepted: 14 April 2016, Published online: 25 August 2016

(C) Ordu University Institute of Health Sciences, Turkey, 2016

\begin{abstract}
Objective: We aimed to evaluate the efficacy of conventional cytology on detecting precancerous lesions in postmenopausal women by comparing the results of colposcopic biopsies of the pre and postmenopausal women with abnormal cervical cytologies.

Methods: Between January 2010-December 2014 we reviewed patients who underwent colposcopic examination in clinic of obstetrics and gynecology of Bakirkoy Dr Sadi Konuk Teaching and Research Hospital retrospectively. The women were evaluated according to menopausal status, abnormal cervical cytologies and colposcopic examination results. NCSS (Number Cruncher Statistical System) for statistical analysis was used.

Results: Patient population ( $\mathrm{n}=1658)$ was composed of 1289 premenopausal $(77.7 \%)$ and 369 postmenopausal $(22.3 \%)$ women. According to the results of cervical cytologies; benign Atypical squamous cells-unknown significance (ASCUS), Atypical squamous cells where a high-grade lesion cannot be eliminated (ASC-H) were not found statistically significant between two groups ( $>0.05)$. Low-grade squamous intraepithelial lesion (LSIL); in premenopausal group, and high-grade squamous intraepithelial lesion (HSIL) in the postmenopausal group were found significantly higher (respectively $\mathrm{p}=0.006$; $\mathrm{p}=0.002 ; \mathrm{p}<0.01)$. When colposcopic results were evaluated; benign findings in postmenopausal women and cervical intraepithelial neoplasia (CIN) I in premenopausal women were found significantly higher (respectively, $\mathrm{p}=0.001 ; \mathrm{p}=0.001 ; \mathrm{p}<0.05$ ). When postmenopausal patients with ASCUS cytology were evaluated, benign biopsy rate was significantly higher in colposcopy, on the other hand, CIN I ratio was significantly higher in premenopausal group with ASCUS cytology $(\mathrm{p}=0.007, \mathrm{p}<0.01)$. When patients with LSIL were evaluated, benign biopsy rate in colposcopy was higher in postmenopausal patients, CIN I and CIN II-C III- Squamose cell carsinoma (SCC) rates were found in highly significant in premenopausal patients $(\mathrm{p}=0.032 ; \mathrm{p}<0.05)$.

Conclusion: When compared with the premenopausal patients group, we have reached the conclusion that the conventional cytology has less efficiency in detecting the precancerous lesions in postmenopausal cases; therefore, colposcopic examination may be appropriate in postmenopausal women. Routine liquid based cytology and HPV screening can achieve clarity in this debate, screening programs should be implemented effectively especially in postmenopausal group and further large scale studies are needed.
\end{abstract}

Key words: Cervical cytology, Menopause, Colposcopy, Precancerous cervical lesions

Address for correspondence/reprints:

Keziban Doğan

Telephone number: +905356336207

E-mail: drkzbn@yahoo.com

DOI: $10.19127 / \mathrm{mbsjohs.76032}$

This research submitted as a oral presentations to Antalya 2016 XI. Turkish German Gynecology Congress.

\section{Introduction}

Cervical cancer is the second most common cancer in women all over the world. Approximately 500 thousand new cases are diagnosed every year; $83 \%$ of them are seen in developing countries. When compared with the previous years, thanks to effective screening programs for the cervical cytology especially in developed countries, the incidence has decreased significantly with early diagnosis and treatment, but it has been continuing 
to be an important cause of morbidity and mortality among women (Parkin et al., 2005, Sankaranarayanan et al., 2005). Although the possible sampling errors and pathological evaluation; the conventional cytology is still the most commonly used method of cervical cancer screening, as it is cheap, easy and easily acceptable by the patients. One of the best ways to demonstrate the effectiveness of the screening is to evaluate the correlation between cervical biopsy and cervical cytology (Rohr et al., 1990). In addition, while it has been reported that the postmenopausal women with abnormal cervical cytology can be monitored in the same way with the general population, the biopsy directed by colposcopy is considered as gold standard in the evaluation of cervical lesions (Massad et al., 2013). The studies on this subject are usually focused on the young patients but the studies for the group of postmenopausal women are few and inadequate (Teaff et al., 1990; Ferenczy et al., 1997, Flynn et al., 2001; Elter et al., 2004). On the other hand, it has been suggested that the abnormal cervical cytology may be inadequate for predicting the precancerous lesions in the postmenopausal women (ASCUS-LSIL Triage Study Group, 2003, Wright et al., 2006). There is no consensus on screening programs which will be held for the detection and management of cervical precancerous lesions in postmenopausal women. In the light of these information; we aimed to evaluate the effectiveness of the conventional cytology on detecting precancerous lesions for the postmenopausal patients by comparing the colposcopic examination and biopsy results with the smear results of the pre-and postmenopausal women with abnormal cervical cytology.

\section{Material and Methods}

Between January 2010-December 2014 a total of 1658 patients taken into colposcopic examination were evaluated retrospectively after receiving of the ethical approval in our hospital which is a tertiary center. The $1289(77.7 \%)$ of the women included in the study were pre-menopausal and $369(22.3 \%)$ of them were post-menopausal. The age, parity, genital warts and smoking history were recorded by examining the digital information in the colposcopy unit. People who use oral contraceptives or hormone replacement therapy, people who have CIN, atypical glandular cells (AGC) and cervical cancer story and also the patients who have endocervical Curettage due to inadequate colposcopy were excluded from the study. The conventional cervical smears were taken with aMedbar ${ }^{\circledR}$ smear brush (Medbar Medical Equipments Tourism Industry Trading Limited Company in Izmir, Turkey) and spread on lama and then sent to the pathology laboratory of our hospital after being fixed with alcohol. The preparations stained with the Papanicolaou method were evaluated according to Bethesda system histologically. The patients with postcoital bleeding, patients who are clinically suspicious of the lesions or cervical erosion in the vaginal examination and also all of the patients directed to the colposcopy examination as they were detected with ASCUS, ASC-H, LSIL, HSIL were included in the study. In the colposcopic examination, the cervix was scanned by small magnification after being washed with saline solution, and then we waited for 60 seconds after the application of 3-5\% acetic acid. The acetowhite areas were scanned by small and large magnifiers and abnormal vascularisations were evaluated with green filter. All of the squamocolumnar junction observed cases were evaluated as satisfactory; unobserved ones were evaluated as unsatisfactory colposcopy. The presence of iodinerepellent area scanned by the application of iodine solution. The biopsies were taken from the suspicious lesions, the regions where abnormal vascularisation, punctuation and mosaicism seen, aseto-white andlugol negative areas. All biopsy specimens were sent to the pathology laboratory for the histopathological examination by being identified in formaldehyde. The results were evaluated in three groups such as the cases which cannot be biopsied or the cases which were evaluated as benign as a result of the biopsy (cervicitis and regenerative changes associated with inflammation, atrophy, cervical polyp, metaplasia), CIN I/mild dysplasia detected cases; CIN II/ moderate dysplasia- CIN III / severe dysplasia squamous cell carcinoma (SCC) detected cases NCSS (Number Cruncher Statistical System) 2007 (Kaysville, Utah, USA) program was used for statistical analysis. In the assessment of research data The Pearson's chi-square test and Fisher Freeman Halton test were used for the comparison of quantitative data in addition to the descriptive statistical methods (Mean, standard deviation, median, frequency and percentage). The results were evaluated at $95 \%$ confidence interval, at the 
$\mathrm{p}<0.05$ level of significance.

\section{Results}

1658 colposcopy performed cases whose demographic characteristics were presented in Table I were evaluated according to menopausal status. 1289 women in the study were pre- menopausal (77.7\%) and $369(22.3 \%)$ were postmenopausal. While the parity was statistically significantly higher in postmenopausal patients, any differences between two groups have been detected in terms of genital warts, smoking, cervical lesions, postcoital bleeding and a history of abnormal cervical cytology.

Table1: The Demographic Characteristics of Cases who Underwent Colposcopic Evaluation

\begin{tabular}{|c|c|c|c|c|}
\hline & $\begin{array}{l}\text { Premenopause } \\
(n=1289,77.7 \%)\end{array}$ & $\begin{array}{l}\text { Postmenopause } \\
(n=369,22.3 \%)\end{array}$ & $\begin{array}{l}\text { Total } \\
(n=1658)\end{array}$ & ${ }^{a}$ p-value \\
\hline$\overline{\text { Age (average } \pm \text { SD) }}{ }^{b}$ & $36.3 \pm 6.7$ & $53.1 \pm 7.1$ & $40.21 \pm 9.68$ & $0.0001 * *$ \\
\hline Parity $^{b}$ & $2(0-5)$ & $3(0-7) *$ & $2(0-7)$ & $0.0001 * *$ \\
\hline Genital warts $(\%)^{\mathbf{b}}$ & $20(1.6)$ & $3(0.8)$ & $23(1.4)$ & 0.285 \\
\hline Smoking (\%) ${ }^{\mathrm{b}}$ & $121(9.4)$ & $32(8.7)$ & $153(9.2)$ & 0.676 \\
\hline Postcoital bleeding $(\%)^{\mathrm{b}}$ & $113(8.8)$ & $32(8.7)$ & $145(8.7)$ & 0.955 \\
\hline Cervical lesions $(\%)^{\mathbf{b}}$ & $351(27.3)$ & $118(32)$ & $469(28.3)$ & 0.174 \\
\hline Abnormal cervical cytology $(\%)^{\mathrm{a}}$ & $805(62.5)$ & $216(58.5)$ & $1021(61.6)$ & a0.173 \\
\hline
\end{tabular}

Table 2: Cytologic and Colposcopic Evaluation Results

\begin{tabular}{llllll}
\hline & & $\begin{array}{c}\text { Premenopause } \\
(\mathbf{n = 1 2 8 9 , 7 7 . 7} \%)\end{array}$ & $\begin{array}{c}\text { Postmenopause } \\
(\mathbf{n = 3 6 9 , 2 2 . 3} \%)\end{array}$ & $\begin{array}{l}\text { Total } \\
(\mathbf{n = 1 6 5 8})\end{array}$ & p-value \\
\hline \hline $\begin{array}{l}\text { Cytologic } \\
\text { evaluation }\end{array}$ & Benign & $432(33.5)$ & $129(35)$ & $562(33.9)$ & 0.493 \\
& ASCUS & $582(45.2)$ & $155(42)$ & $737(44.5)$ & 0.284 \\
& ASC-H & $30(2.3)$ & $14(3.8)$ & $44(2.7)$ & 0.122 \\
& LSIL & $207(16.1)^{*}$ & $38(10.3)$ & $245(14.8)$ & $0.006 * *$ \\
& HSIL & $44(3.5)$ & $26(7.1)^{*}$ & $70(4.2)$ & $0.002 * *$ \\
\hline $\begin{array}{l}\text { Colposcopic } \\
\text { evaluation }\end{array}$ & Benign-no biopsy & $845(65.6)$ & $276(74.8)^{*}$ & $1121(67.6)$ & $0.001 * *$ \\
& CIN I & $334(25.9)^{*}$ & $60(16.3)$ & $394(23.8)$ & $0.001 * *$ \\
& CIN II-III-SCC & $110(8.5)$ & $33(8.9)$ & $143(8.6)$ & 0.805 \\
\hline
\end{tabular}

ASCUS ; Atypical squamous cells - unknown significance, ASC-H;Atypical squamous cells where a high-grade lesion cannot be eliminated,LSIL; Low-grade squamous intraepithelial lesion, HSIL; High-grade squamous intraepithelial lesion, CIN;Cervical intraepithelial neoplasia. SCC; Squamose cell carsinoma

Tablo 3: The Relationship Between Cervical Cytology and Colposcopy Findings

\begin{tabular}{lllllll}
\hline & \multirow{2}{*}{ Colposcopy } & \multicolumn{4}{c}{ Cervical cytology } \\
\cline { 3 - 7 } Premenopause & Benign, nobiopsy & $354(83,1)$ & $373(64,1)$ & $10(33,3)$ & $101(48,8)$ & $7(16,7)$ \\
& CIN I & $62(14,6)$ & $169(29) *$ & $9(30)$ & $85(41,1)^{*}$ & $9(21,4)$ \\
& CIN II\&III\&SCC & $10(2,3)$ & $40(6,9)$ & $11(36,7)$ & $21(10,1)^{*}$ & $26(61,9)$ \\
& Total & $426(100)$ & $582(100)$ & $30(100)$ & $207(100)$ & $42(100)$ \\
\hline Postmenopause & Benign, nobiopsy & $113(87,6)$ & $120(77,4)^{*}$ & $5(35,7)$ & $27(71,1)^{*}$ & $5(20)$ \\
& CIN I & $14(10,9)$ & $28(18,1)$ & $1(7,1)$ & $10(26,3)$ & $6(24)$ \\
& CIN II\&III\&SCC & $2(1,6)$ & $7(4,5)$ & $8(57,1)$ & $1(2,6)$ & $14(56)$ \\
& Total & $129(100)$ & $155(100)$ & $14(100)$ & $38(100)$ & $25(100)$ \\
\hline
\end{tabular}

${ }^{\mathrm{a}}$ Pearson Ki-kare test

${ }^{\mathrm{b}}$ Fisher Freeman Halton test $* \mathrm{p}<0.05 * * \mathrm{p}<0.01$ 
Smear and colposcopy results were presented in Table II. According to the smear results; while any statistically significant differences were found between the groups $(p>0.05)$ in terms of benign, ASCUS, ASC-H distributions, in premenopausal group the LSIL, in the postmenopausal group the HSIL were found to be statistically significantly higher (respectively, $\mathrm{p}=0.006 ; \mathrm{p}=0.002 ; \mathrm{p}<0.01$ ).

When colposcopy results were evaluated; benign findings in postmenopausal women; CIN I in the premenopausal group were determined to be statistically significantly higher (respectivelyp $=0.001 ; \mathrm{p}=0.001 ; \mathrm{p}<0.05$ ).

In terms of the group consisting of CIN II, CIN III and SCC no significant difference was detected according to the menopausal status $(\mathrm{p}=0.805$, $\mathrm{p}>0.05$ ).

The relationship between smear results and colposcopy findings have been presented in Table III. According to menopausal status, there was no statistically significant difference between the distribution of colposcopy results of the cases whose smears have bening, ASC-H and HSIL (respectively, $\mathrm{p}=0.468, \mathrm{p}=0.223, \mathrm{p}=0.889 \mathrm{p}>0,05$ ). When colposcopy results were evaluated according to menopausal status, statistically significant differences were found in the cases who have ASCUS and LSIL in smear. When the significance was analyzed in terms of ASCUS, CIN I ratio in premenopausal group was significantly higher as benign biopsy rate was high in the colposcopies of postmenopausal patients $(\mathrm{p}=0.007 ; \mathrm{p}<0.01)$. When the significance was analyzed in terms of LSIL, while the normal biopsy rate was high in colposcopy of postmenopausal patients, CIN I and CIN II-CIN III-SCC colposcopy finding rates of the patients without menopause were found to be significantly high $(\mathrm{p}=0.032 ; \mathrm{p}<0.05)$. While the ASCUS / LSIL ratio was 4:07 in postmenopausal women, we have found it as 2.8 in premenopausal group.

\section{Discussion}

At the ASCCP (American Society for Colposcopy and Cervical Pathology) consensus presented in 2012, it has been reported that the postmenopausal women with abnormal cervical cytology can be followed in the same way with the general population and after 65 years old there is no need to scan for the women who have no history of abnormal cervical cytology and previously regular screening (Massad et al., 2013). Compared to the young women, effective cervical cancer screening programs have not been implemented for postmenopausal women depending on genital atrophy, therefore it has limited the diagnostic methods such as colposcopy and smear, because of these reasons the risk of invasive cervical cancer have been continued to increase with age (Sellors et al., 2002, National Cancer Institute Workshop, 1993). The hormonal changes developing in the postmenopausal period, especially hypoestrogenism situation causes atrophies of the genital organs, and atypical findings in cervical cytology. Again the possibilities of inadequate colposcopic examination have been increased as the transformation zone can't be evaluated due to the genital atrophy (Dresang LT, 2005). Therefore, we have not included the cases with inadequate colposcopy findings in our study. Furthermore, the benign degenerative changes in the immature squamous cells connected to the hypoestrogenism, obvious atrophy can imitate squamous intraepithelial lesions and even invasive cancer in postmenopausal women (Saad et al., 2006). Unlike young women, the growth in squamous cell nucleus is a histological finding often seen inpostmenopausal women. Especially the growth of squamous cell nucleus has been increased the diagnosis of ASCUS in postmenopausal cases and compared to young patients ASCUS/LSIL rate has been increasing (Selvaggi et al., 2002; Massadet al., 2003; Saad et al., 2006). While the ASCUS/LSIL ratio of postmenopausal patientswas 4.07 , we have found it as 2.8 for the premenapausal group. Even if the relieving of the genital atrophy with estrogen therapy in postmenopausal women is considered as a suitable method, this subject is also controversial. As there are studies suggesting that local estrogen therapy can distinguish thereal preneoplastic changes with benign cytologies imitating atrophy by decreasing vaginal atrophy (Piccoli et al., 2008), there are also studies suggesting that the hormone replacement therapy (HRT) can cause the artifacts similar to the cytology findings imitating LSIL by increasing the glycogenation (Jemal et al., 2004). While local estrogen therapy was recommended for the postmenopausal women in the 2006 ASCCP consensus, this proposal was withdrawn in 2012 (Massad et al., 2013). Because of this discussion, we have excluded the cases using HRT or local estrogen from the study. In our study, the LSIL in premenopausal group; HSIL in postmenopausal group were determined significantly high. 
When colposcopy results were evaluated, benign findings in postmenopausal women; CIN I in the premenopausal group were found to be significantly high. These findings were considered because of progression of which a part of the preinvasive cervical lesions showed with age or regression the majority suffered from (Melnikow et al., 1998, Bansal et al., 2008). Considering the relationship between the results of smear and colposcopy findings; while benign biopsy rate was statistically higher at colposcopy in the postmenopausal cases whose smear result was ASCUS, CIN I rate was significantly higher in premenopausal group. Similarly, when the significance examined in the cases where LSIL detected as a result of the smear, while benign biopsy rate was high at colposcopy in postmenopausal group, CIN I and CIN II-III-SCC rates were found significantly high for the nonmenopausal women. There are studies suggesting that the abnormal cervical cytology predicts the precancerous cervical lesions less frequently in postmenopausal women than premenopausal women due to epithelial changes in atrophy (Kobelinet al., 1998; Sawaya et al., 2000; Keatinget al., 2001; ASCUS-LSIL Triage Study Group, 2003; Wright et al., 2006). In our study also, the abnormal cervical cytology findings including ASCUS and LSIL are inadequate for predicting the precancerous lesions in postmenopausal women in comparison with the premenopausal patients. It is known that postmenopausal women were less likely to apply for a gynecological examination because of traditional beliefs in our country. Considering the failure in application of the effective cervical cancer screening programs in postmenopausal women for these findings, it will be appropriate to direct the abnormal cervical cytology detected cases to the colposcopy. The limitations of our study are being retrospective, our lack of long-term follow-up cases, and taking of cervical cytology with the conventional method. In addition, as it was not for routine use, HPV screening could not be made. When the liquid-based techniques which have been entering into a routine use recently in our country and the data which will be obtained by HPV screening are evaluated, we can reach more reliable results.

\section{Conclusion}

When compared with premenopausal patients the conventional cytology seems to be less effective in detecting the precancerous lesions for postmenopausal cases. The benefits of hormone replacement therapy is controversial because of different operational problems. The data which will be obtained with the entry ofthe liquid-based cytology and HPV screening into the routine clinical practicewill enable us to achieve more reliable results. In addition, without any neglect, more effective implementation of screening programs especially in postmenopausal patients and further studies are needed for theclarification of this issue.

Informed Consent: Necessary information using the patient information form and consent form was taken from the patients.

Peer-review: Externally peer-reviewed.

Author Contributions: Concept-KD, Design-KD, Supervision-HK, Funding-KD, Data Collection and/or Processing-KD, Literature Review-KD, Writing-KD, Critical Review-KD.

Conflict of Interest: No conflict of interest was declared by the authors.

Financial Disclosure: The authors declared that this study hasn't received any financial support.

\section{References}

ASCUS-LSIL Triage Study (ALTS) Group. Results of a randomized trial on the management of cytology interpretations of atypical squamous cells of undetermined significance. Am J Obstet Gynecol 2003; 188: 1383-92.

Bansal N, Wright JD, Cohen CJ, Herzog TJ.Natural history of established low grade cervical intraepithelial (CIN 1) lesions. Anticancer Res 2008; 28: 17-63.

Dresang LT. Colposcopy: an evidence-based update. J Am Board Fam Pract 2005; 18: 383-92.

Elter K, Durmusoglu F, Sezen D, Uygur M. The positive predictive value of annual cervical smears in postmenopausal women. Turkiye Klinikleri J Gynecol Obst 2004; 14: 311-6.

Ferenczy A, Gelfand MM, Franco E, Mansour N. Human papillomavirus infection in postmenopausal women with and without hormone therapy. Obstet Gynecol 1997; 90: 7-11. 
Flynn K, Rimm DL. Diagnosis of ASCUS' in women over age 50 is less likely to be associated with dysplasia. Diagn Cytopathol. 2001; 24: 1326.

Jemal A, Tiwari RC, Murray T, Ghafoor A, Samuels A, Ward E. Cancer statistics, 2004. Cancer J Clin 2004; 54: 8-29.

Keating JT, Wang HH. Significance of a diagnosis of atypical squamous cells of undetermined significancefor Papanicolaou smears in perimenopausal and postmenopausal women.Cancer . 2001; 93:100-105.

Kobelin MH, Kobelin CG, Burke L, Lavin P, Niloff JM, Kim YB. Incidence and predictors of cervical dysplasia in patients with minimally abnormal Papanicolaou smears.Obstet Gynecol. 1998; 92: 356-359.

Massad LS, Behbakht K, Collins YC, Cejtin HE. Histologic findings from the cervix among older women with abnormal cervical cytology. Gynecol Oncol. 2003; 88(3): 340-344.

Massad LS, Einstein MH, Huh WK, et al.2012 updated consensus guidlines for management of abnormal cervical screening tests and cancer precursors. J Low Genit Tract Dis. 2013; 17: 1.

Melnikow J, Nuovo J, Willan AR, Chan BK, Howell LP. Natural history of cervical squamous intraepithelial lesions: a meta-analysis. Obstet Gynecol. 1998; 92(4 Pt 2): 727-35.

National Cancer InstituteWorkshop. The Bethesda System for reporting cervical/vaginal cytologic diagnoses: revised after second National Cancer Institute Workshop (April 29-30, 1991). Acta Cytol 1993; 37: 115-24

Parkin DM, Bray F, Ferlay J, Pisani P: Global cancer statistics, 2002. CA Cancer J Clin 2005, 55:74-108
Piccoli R, Mandato VD, Lavitola G, et al. Atypical squamous cells and low squamous intraepithelial lesions in postmenopausal women: implications for management. Eur J Obstet Gynecol Reprod Biol. 2008; 140(2): 269-274.

Rohr LR: Quality assurance in gynecologic cytology. What is practical? Am J Clin Pathol 1990; 94:754-758

Saad R, Dabbs D, Kordunsky L, et al. Clinical significance of cytologic diagnosis of atypical squamous cells, cannot exclude high grade, in perimenopausal and postmenopausal women. Am J Clin Pathol. 2006; 126(3): 381-388.

Saad R, Kanbour-Shakir A, Lu Erxiong, Modery J, Kanbour A. Cytomorphologic analysis and histological correlation of high grade squamous intraepithelial lesions in postmenopausal women. Diagn Cytopathol. 2006; 34(7): 467-471.

Sankaranarayanan R, Gaffikin L, Jacob M, Sellors J, Robles S: A critical assessment of screening methods for cervical neoplasia. Int $\mathbf{J}$ Gynaecol Obstet. 2005; 89: 4-12

Sawaya GF, Grady D, Kerlikowske K, et al. The positive predictive value of cervical smears in previously screened postmenopausal women: the heart and estrogen/progestin replacement study (HERS). Ann Intern Med. 2000; 133(12): 942950. 